\title{
Study of Chronic Calcific Pancreatitis: 30 Cases
}

Mohammad Saief Uddin ${ }^{1 *}$, Mohammad Abul Kalam Azad $^{2}$, Sarwar Ahmed Sobhan ${ }^{3}$, Md Asaduzzaman Nur ${ }^{4}$, Md Zulfiqur R Khan

\footnotetext{
${ }^{1}$ Assistant Professor, Department of Hepatobiliary, Pancreatic and Liver Transplant Surgery, Bangabandhu Sheikh Mujib Medical University, Shahbag, Dhaka, Bangladesh

${ }^{2}$ Medical Officer, Department of Hepatobiliary, Pancreatic and Liver Transplant Surgery, Bangabandhu Sheikh Mujib Medical University, Shahbag, Dhaka, Bangladesh

${ }^{3}$ Medical Officer, Department of Hepatobiliary, Pancreatic and Liver Transplant Surgery, Bangabandhu Sheikh Mujib Medical University, Shahbag, Dhaka, Bangladesh

${ }^{4}$ Assistnt Professor, Department of Surgery, Enam Medical College Dhaka, Bangladesh

${ }^{5}$ Professor \& Chairman, Department of Hepatobiliary, Pancreatic and Liver Transplant Surgery, Bangabandhu Sheikh Mujib Medical University, Shahbag, Dhaka, Bangladesh
}

DOI: $10.36347 /$ sjams.2020.v08i09.036

| Received: 14.09 .2020 | Accepted: 24.09.2020 | Published: 26.09 .2020

*Corresponding author: Dr. Mohammad Saief Uddin

Abstract

Original Research Article

Background: The clinical course of chronic pancreatitis is still unpredictable, which relates to the lack of the availability of a clinical classification. Chronic calcific pancreatitis is a special form of chronic pancreatitis that tends to calcify or is associated with pancreatic lithiasis. Information regarding the presentations, physical findings, diagnostic findings and complications are very important for deciding about the treatment approach for calcific pancreatitis. Aim of the study: The aim of this study was to collect information regarding the presentations, physical findings, diagnostic findings complications the patients with chronic calcific pancreatitis. Methods: This prospective observational study was conducted in the Department of Hepatobiliary, Pancreatic and Liver Transplant Surgery, Bangabandhu Sheikh Mujib Medical University, Shahbag, Dhaka, Bangladesh during the period from January 2017 to December 2017. Thirty (30) patients of pancreatic calculi were studied in this study. All the data were collected by a pre-designed questioner, analyzed by SPSS version 20 and findings were displayed by several tables and figures of MS Office program. Result: In total 27 participants were male and 3 were female. So the male-female ratio was 9:1. All the patients of this series presented with abdominal pain (100\%). Weight loss presented in 10 (33.33\%), Steatorrhoea in $9(30 \%)$, abdominal lump in $4(13.33 \%)$ \& diabetes in (46.67\%) patients. Jaundice was present in 3 cases $(10 \%)$. Palpable lump in the epigastric region in 5 cases $(16.67 \%)$ and tenderness in epigastric region in 10 patients $(33.33 \%)$. Oedema was presented by 4 patients $(13.33 \%)$, Ascites presented by 7 patient (23.33), Palpable liver was in 1 patient (3.33\%) and palpable gall bladder in 2 patient (6.67\%). As complication 30 patients 2 patients (6.67) developed wound infection in the post-operative period, 2 patients $(6.67 \%)$ developed respiratory tract infection one patient $(3.33 \%)$ developed pancreatic fistula, one patient $(3.33 \%)$ developed sub-acute intestinal obstruction and one patient $(3.33 \%)$ developed jaundice. All are treated conservatively. No patient died in the post-operative period in hospital. Conclusion: This study carries some epidemiological significance in certain aspects. Persistent or recurrent pain is the most common presentation in this study. In diagnosis history of pain, radiology and Ultrasonography were used. CT scan and ERCP is the second line investigation in the diagnostic procedures. If indicated most of the cases are operable and drainage procedure (Puestow procedure) is the best option. Surgery is a standard form of treatment and outcome of surgery is quite appreciable in our country except few cases which histologically proved malignant postoperatively and later was treated by chemotherapy.

Keywords: Pancreas, Features, Chronic calcific pancreatitis, GI tract, Digestive system.

Copyright @ 2020: This is an open-access article distributed under the terms of the Creative Commons Attribution license which permits unrestricted use, distribution, and reproduction in any medium for non-commercial use (NonCommercial, or CC-BY-NC) provided the original author and source are credited.

\section{INTRODUCTION}

The clinical course of chronic pancreatitis is still unpredictable, which relates to the lack of the availability of a clinical classification. Chronic calcific pancreatitis is a special form of chronic pancreatitis that tends to calcify or is associated with pancreatic lithiasis.
Information regarding the presentations, physical findings, diagnostic findings and complications are very important for deciding about the treatment approach for calcific pancreatitis. Because of the lack of normal pancreatic enzymes, digestion of food and the production of wastes are adversely affected. Abdominal pain is common, especially after eating. Stools become 
bulky, greasy, foul smelling and tend to float in the water because of their high fat content - a condition known as steatorrhea. The formation of stones in the pancreas is also common. The treatment of chronic pancreatitis depends on 4 factors: the cause of the pancreatitis, the portion of the pancreas involved the presence or absence of symptoms, and the size of the pancreatic duct. When symptoms are mild or absent ("silent pancreatitis") no treatment is indicated. For persons with disabling symptoms, however, treatment is indicated. The main goal of treatment is to provide sufficient and lasting pain relief without the use of opoid and to prevention of deteriorations of pancreatic function. Treatment may consist of medications and surgery. Pancreatic calculi or calcific pancreatitis are uncommon. They consist of calcium carbonate and little phosphate [1]. They are not the primary disease of the pancreas but are secondary to inflammatory pancreatic diseases. Stones are found in the ducts and rarely in the interstitium, vary from concretions invisible to the naked eye to one centimeter to several centimeters in diameter in association with chronic pancreatitis. Pancreatic inflammatory disease presents, clinically and histologically, as a spectrum of disorders at one end of the spectrum is the acute pancreatitis and at the other is the chronic pancreatitis and classified by Marseilles, France in 1963 as follows [2,3]: 'acute Pancreatitis means single attack of pancreatitis is a previously normal gland', 'acute relapsing pancreatitis means recurrent attacks of acute pancreatitis of moderate severity, without permanent damage to the Pancreas', 'Chronic Relapsing pancreatitis - means frequent attacks of pancreatitis leading to permanent damage to the gland evidenced by altered pancreatic function, pancreatography, operative exploration and biopsy' and chronic pancreatitis-means frequent to continuous attacks of pancreatitis leading to scarring, severe fibrosis, intermittent ductal obstruction and dilatation. Pancreatic calcification is seen radio graphically; steatorrhoea reveals pancreatic insufficiency and diabetes mellitus is frequently found. In approximately $80-85 \%$ of patients a cause can be identified in $15-20 \%$ cases no cause is apparent and the disease is idiopathic [4]. The causes are alcoholism, biliary tract diseases, trauma, hereditary pancreatitis, infections, metabolic dysfunction, drugs, connective tissue disorder and vasculitis, penetrating duodenal ulcer and/or obstruction of the ampulla of Vater. The basic process of pathogenesis of pancreatitis is the auto digestion of the pancreas following activation of the enzymes within the gland. There are many hypotheses regarding the activation of the pro-enzymes such as [5] acinar cell injury (Virus, endotoxins, toxic chemicals and trauma result is direct acinar cell damage, with intrapancreatic activation and release of enzymes. It is the currently favored theory rather than alcoholism and biliary tract disease), duct obstruction (Duct obstruction due to impaction of gallstones in the ampulla of vater results in increase intraductal pressure), hyperlipidemia (Hyperlipoproteinemia (type 1 and V), oestrogen therapy, acute alcoholism raise serum triglycerides (more than $1000 \mathrm{mg} / \mathrm{dl}$ ). probably lipolysis of triglycerides by pancreatic lipase in the around the pancreas leads to increased local FFA which is toxic to acinar cells and blood vessels) and hypercalcemia (7\% to $19 \%$ of patients of pancreatitis have either a parathyroid adenoma or a parathyroid carcinoma, probably raised calcium con-centration causes auto activation of trypsinogen). Probably trypsin plays the key role in the activation of the pro-enzymes taking part in the process of auto digestion. Two other enzymes are also very important elastase and phospholipase a are also present in the acinar cells in inactivated form and activated by trypsin. Elastase causes dissolution of elastic fibres of blood vessels and ducts. It is likely the culprit in the production of haemotrhage. Phospholipase A when activated has two effects: destruction of cell membrane and conversion of lecithin in bile to lysolecithin, which is highly toxic and capable of damaging the ductal system of the pancreas. Chronic pancreatitis is rarely preceded by acute pancreatis; but the same aetiological factors are responsible in many cases with special causes like severe protein caloric malnutrition, familial hereditary pancreatitis (Autosomal dominant disease with incomplete penetrance.), pancreas divisum (12\% cases there is incomplete fusion of ventral and dorsal pancreatic anlage), hypercalcemia and hyperlipidemia. Proteincalorie malnutrition probably plays a role in the tropical pancreatitis present in South East Asia and parts of Africa, where alcohol consumption is extremely low. Pancreatic diabetes (Calcific fibrosis of pancreas) is common in some developing countries like Brazil, India (Kerala), Indonesia, Nigeria, Uganda and Zaire. An association between pancreatic damage and the consumption of tapioca (cassava) has been suggested. Tapioca is a tuberous root and has been found to be a major source of dietary cyanide. Other foods like yams, sorghumand millet also contain cyanide but in small quantities. Lack of Sulfur-containing amino acid in the diet or in the body reduces the formation of this thiocyante a major pathway of cyanide detoxification. Therefore, consumption of tapioca or other cyanide containing food stuff by populations with marginal nutrition or malnutrition, if any lead to excessive accumulation of cyanide, resulting in pancreatic damage [6]. Age of the patients may be a potential factor of chronic calcific pancreatitis. In western countries the usual age incidence of pancreatitis is above 40 years though hereditary pancreatitis may occur in the younger age group. In Uganda and Nigeria diabetes associated with pancreatic calculi is often seen below the age of 40. The patients of a long series of 600 cases of Pancreatic calculi by Geevarghese[7] from Kerala state of India showed youngest of a 7 year old girl andthe oldest of an of 62 years. We have tried to dig out the information regarding the overall status of the patients with chronic calcific pancreatitis in this study. 


\section{OBJECTIVES}

General Objective

- To evaluate the status of the patients with chronic calcific pancreatitis.

\section{Specific Objective}

- To evaluate the physical findings among the participants.

- To evaluate the ultrasonographic findings among the participants.

\section{Methodology \& Materials}

This prospective observational study was conducted in the Department of Hepatobiliary, Pancreatic and Liver Transplant Surgery, Bangabandhu Sheikh Mujib Medical University, Shahbag, Dhaka, Bangladesh during the period from January 2017 to December 2017. Thirty (30) patients of pancreatic calculi were studied in this study. Evaluation of the patients included complete history with detailed information about occupation, socio-economic condition, diet, past illness and family history. The diagnosis was established on careful history taking and through clinical examination but radiography and ultrasonography studies played the major role and ERCP also played some definitive role. A thorough physical examination including facies, pulse, respiration, temperature, blood pressure, anaemia, jaundice, Oedema, dehydration, heart, lungs, signs of diabetes mellitus and vitamin deficiency were recorded. The typical facies of pancreatic calculi, bilateral nontender parotid swelling with protuberant belly in an emaciated body was found in one patient. The abdomen was examined for tenderness, rigidity, and distension ascites and palpated for liver, spleen kidneys or any other mass. Laboratory investigations for blood included $\mathrm{Hb} \%$, total and differential count of leukocytes, bleeding time, clotting time, ESR blood urea, fasting blood sugar along with 2 hrs blood sugar, Serum calcium, Serum amylase, Serum bilirubin, serum alkaline phosphate, serum transaminases (SGOT, SGOPT), total protein, serum albumin, serum globulin, serum cholesterol etc. Besides these urine and stool were examined routinely. In one case the ascetic fluid was also biochemically studied. In radiographic examination, plain $\mathrm{X}$-ray of the abdomen (in anteroposterior and lateral view), X-ray chest (Posteroanterior view) done. Ultra-sonographic examination of the hepatobiliary system and pancreas and CT scan done for all patient and ERCP were also done in 15 patients. All patient treated surgically by drainage procedure (Puestow procedure), in 6 cases where suspected malignancy biopsies were taken. All the data were collected by a pre-designed questioner, analyzed by SPSS version 20 and findings were displayed by several tables and figures of MS Office program.

\section{RESULT}

In this study the age range of the participants was 16 to 65 years. The average ages in males and females in the series are 44 and 30 respectively. In total 27 participants were male and 3 were female. So the male-female ratio was 9:1. All the patients of this series presented with abdominal pain (100\%) which started within 1-2 hours of a heavy or a normal meal, few accompanied by nausea, vomiting and loss of appetite. Pain starts suddenly in majority cases in the epigastrium or in either or both hypochondrium passing to the back and ultimately localized in the epigastrium. The pain is not relieved by antacids or usual analgesics, better in empty stomach and leaning forwards or sitting position; worse in lying position or after full meals. The pain persists relentless for one week or so and subsides and again after several months or years comes all on a sudden. Weight loss presented by $10(33.33 \%)$ patient, Steatorrhoea by $9(30 \%)$ patient, abdominal lump by 4 $(13.33 \%)$ patient, 14 patients $(46.67 \%)$ were diabetic and all patient have vague complain of anorexia, nausea \& vomiting. Present study shows that among 30 cases 6 patients $(20 \%)$ were anaemia with evidence of significant weight loss. Jaundice was present in 3 cases $(10 \%)$. Palpable lump in the epigastric region in 5 cases $(16.67 \%)$ and tenderness in epigastric region in 10 patients $(33.33 \%)$. Oedema was presented by 4 patients (13.33\%), Ascites presented by 7 patient (23.33), Palpable liver was in 1 patient $(3.33 \%)$ and palpable gall bladder in 2 patient $(6.67 \%)$. Present study shows that serum alkaline phosphates was raised in 20 patients (66\%). Prothrombin time normal was found in 27 cases (90\%) and prolonged in 03 cases $(10 \%)$ of 30 patients. CT scan findings of this study of 15 cases reveals that pancreatic duct dilatation in 15 patients $(100 \%)$ with stones within duct 30 cases $(100 \%)$. Dilatation of the bile duct in 3 patients (20\%). Associated distended GB in 2 patients (13.67\%). Pancreatic parenchymal change in 12 patients $(80 \%)$, pancreatic duct dilatation with stone in the duct all patient (100\%). Study shows that, ultrasonogram was positive for pancreatic duct dilatation in 30 patients $(100 \%)$ with stones within duct 30 cases $(100 \%)$. Dilatation of the bile duct in 3 patients $(10 \%)$. Associated distended GB in 2 patients $(6.67 \%)$. Pancreatic parenchymal change in 18 patients $(60 \%)$, pancreatic duct dilatation with stone in the duct all patient $(100 \%)$. ERCP was done in 25 cases. All $(100 \%)$ have dilated pancreatic duct with stone. In 3 patient $(12 \%)$ were found dilated CBD which at the same time presented with jaundice. Per-operative finding of this study reveals that stone were present in all cases $(100 \%)$ where ducts were also dilated, in 18 patients $(60 \%)$ stone present in the parenchyma, in 13 cases $(43 \%)$ pancreas were found firm to hard and indurated consistent with chronic pancreatitis but in 6 cases $(20 \%)$ operative findings seems to be malignant so biopsy taken. Present study shows that out of 30 patients 2 patients (6.67) developed wound infection in the post-operative period, 2 patients $(6.67 \%)$ developed respiratory tract infection one patient (3.33\%) 
developed pancreatic fistula, one patient (3.33\%) developed sub-acute intestinal obstruction, one patient (3.33\%) developed jaundice. All are treated conservatively. No patient died in the post-operative period in hospital.

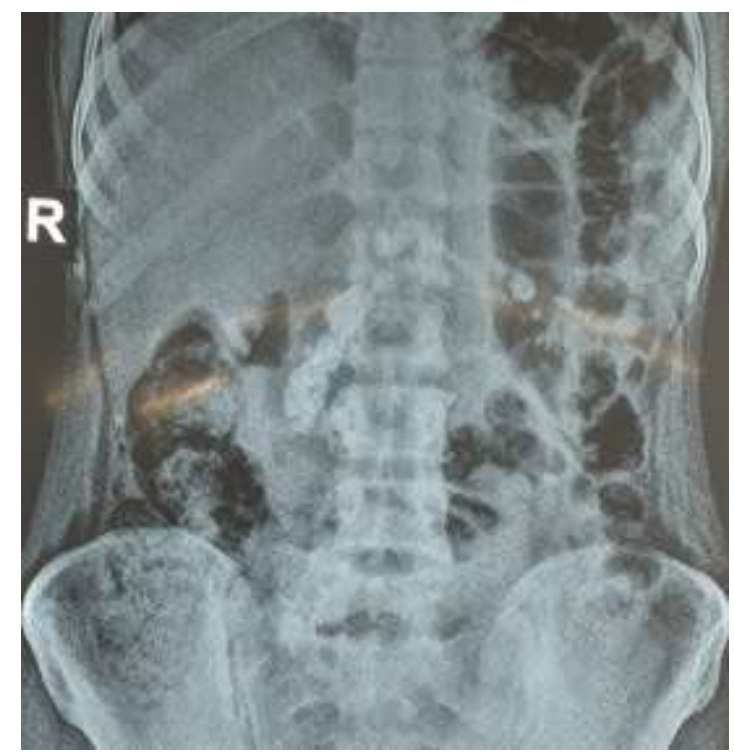

Pancreatic calcification

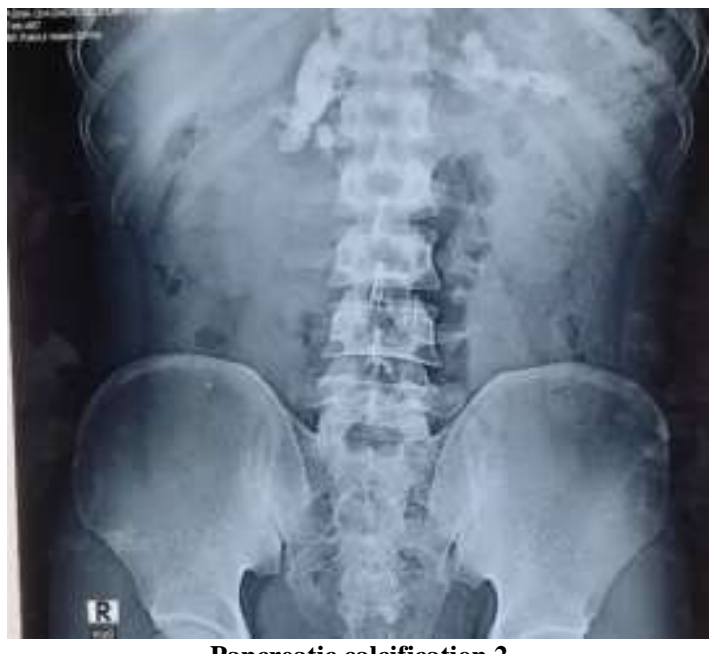

Pancreatic calcification 2

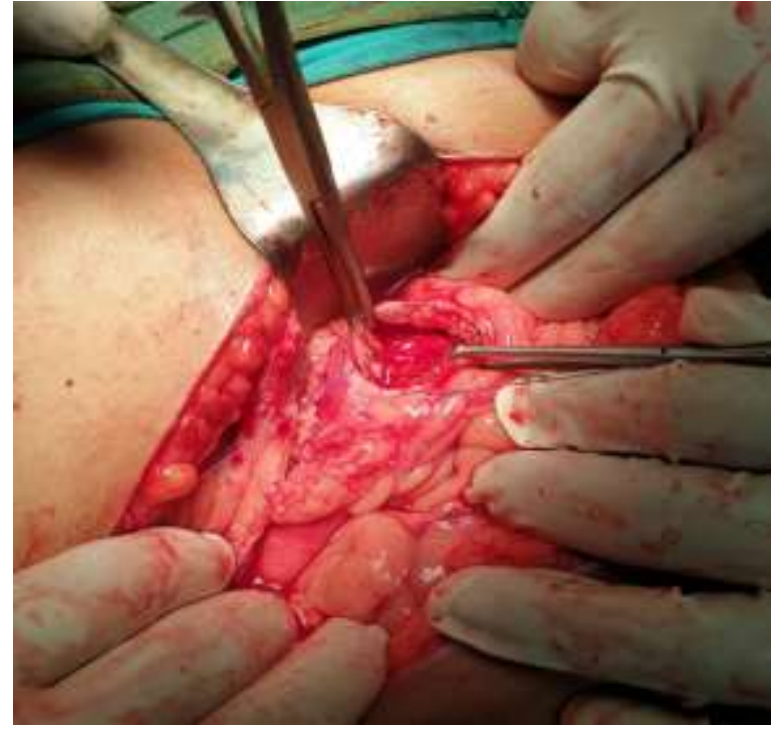

Dilated PD

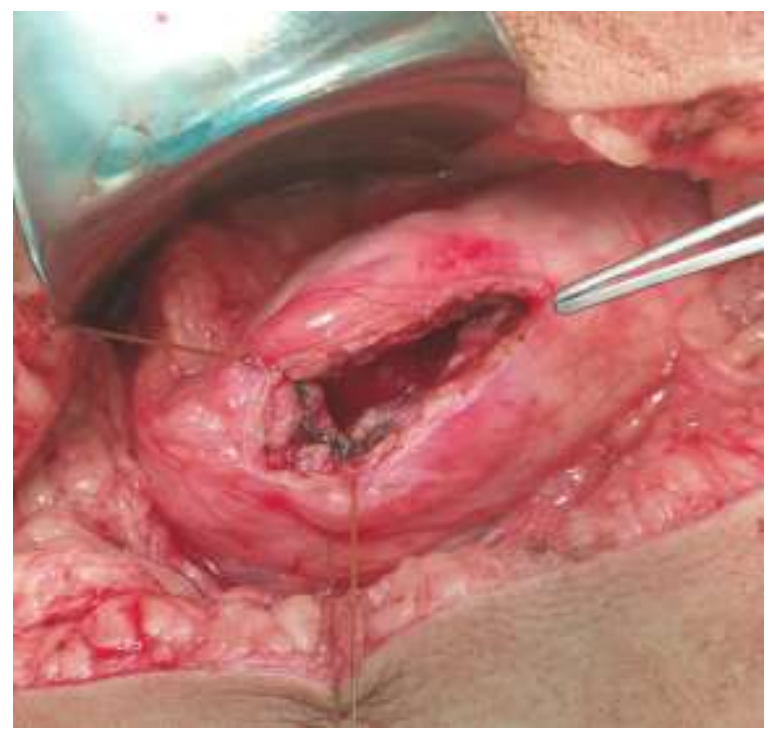

Exploration of PD

Table-I: Age distribution of participants $(n=30)$

\begin{tabular}{|c|c|c|c|}
\hline Age & Male & Female & Total \\
\hline $10-20$ & 1 & 1 & 2 \\
\hline $21-30$ & 3 & 0 & 3 \\
\hline $31-40$ & 6 & 0 & 6 \\
\hline $41-50$ & 9 & 1 & 10 \\
\hline $51-60$ & 7 & 1 & 8 \\
\hline $61-70$ & 1 & 0 & 1 \\
\hline Total & 27 & 3 & 30 \\
\hline$\%$ & $90 \%$ & $10 \%$ & $100 \%$ \\
\hline
\end{tabular}




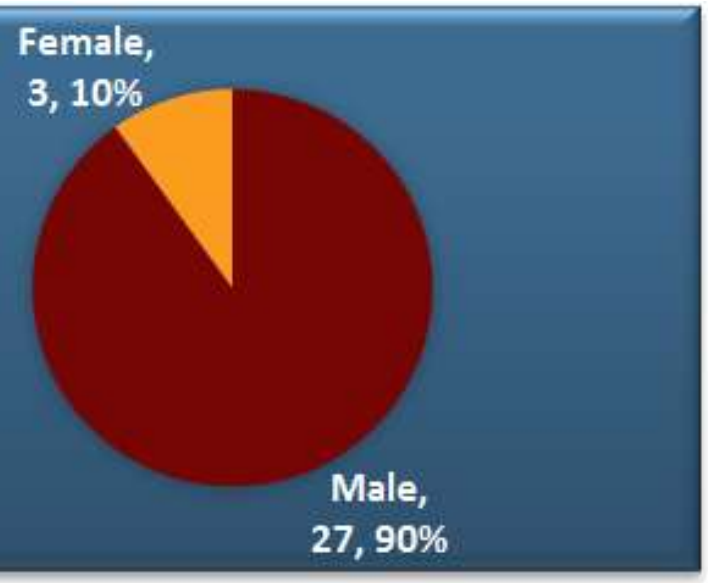

Fig-I: Gender distribution of participants $(n=30)$

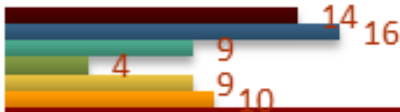

Diabetes malitus $\mathbf{m}$ Smooking

- Alcoholic $\quad$ Lump

- Steatorrhoea $\quad$ Weight loss

- Pain

Fig-II: Distribution of clinical presentations among participants $(\mathbf{n}=30)$

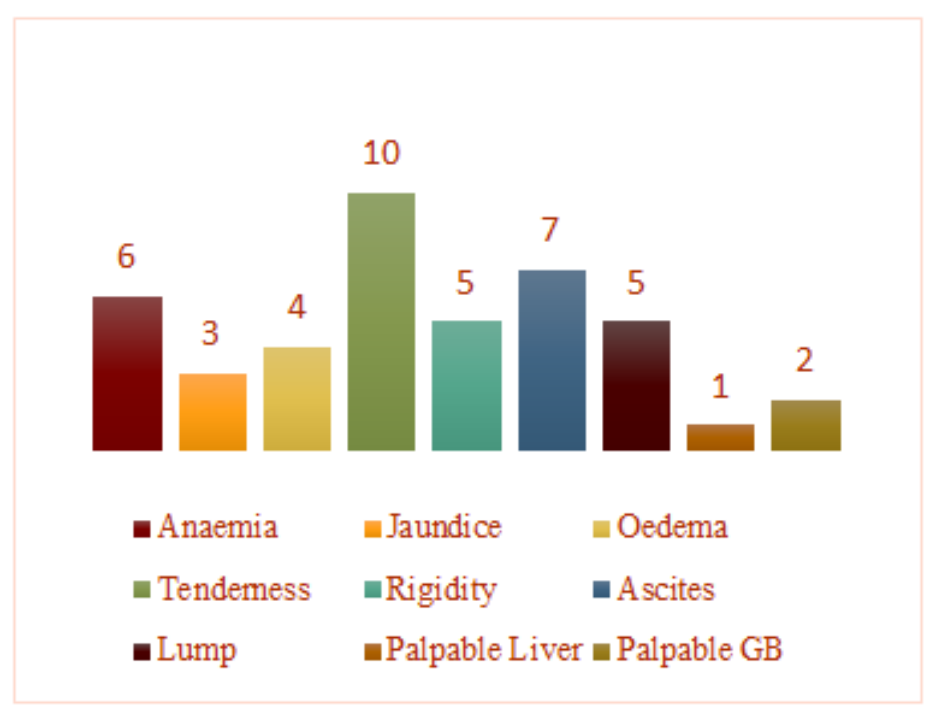

Fig-III: Distribution of physical findings among participants $(\mathbf{n = 3 0})$

Table-II: Ultrasonographic findings of the hepatobiliary \& pancreatic system of participants $(n=30)$

\begin{tabular}{|l|c|c|}
\hline \multicolumn{1}{|c|}{ Findings } & n & $\mathbf{\%}$ \\
\hline Dilatation of CBD & 3 & 10 \\
\hline Distended GB & 2 & 6.67 \\
\hline Pancreatic parenchymal change & 18 & 60 \\
\hline Pancreatic duct dilatation & 30 & 100 \\
\hline Stone in parenchyma & 14 & 46 \\
\hline Stone in the duct & 30 & 100 \\
\hline
\end{tabular}




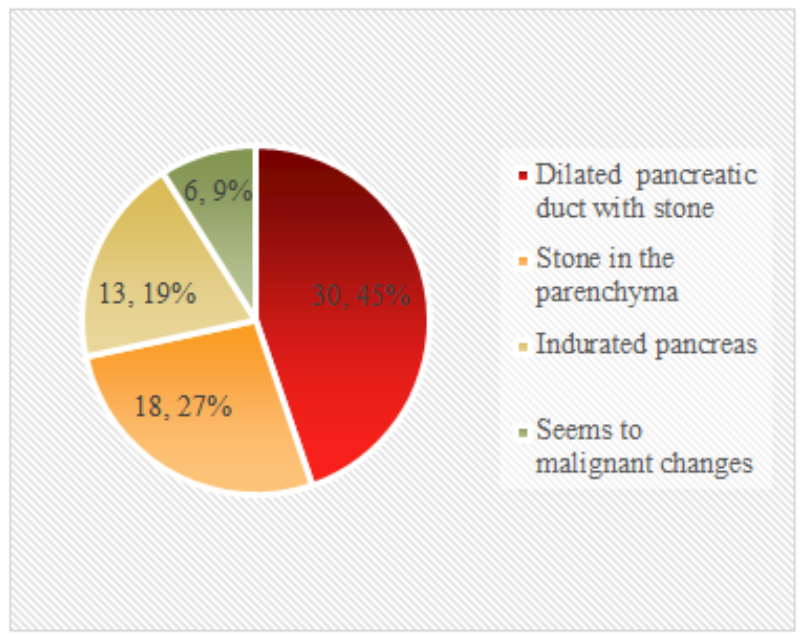

Fig-IV: Per-operative finding among participants $(n=30)$

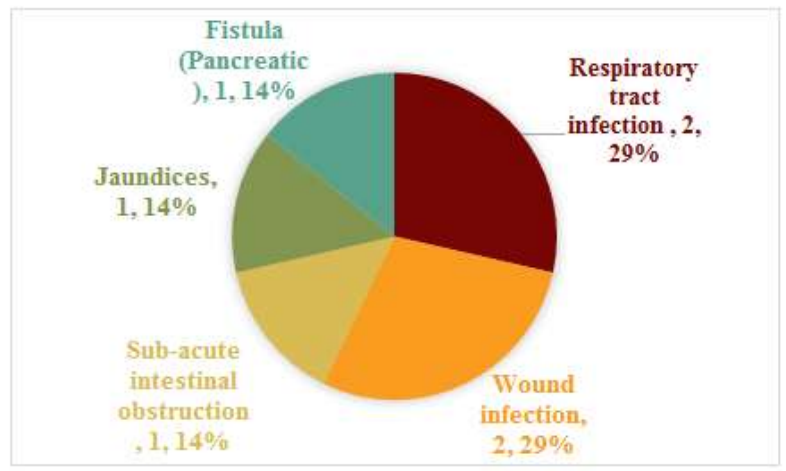

Fig-V: Complications among participants $(n=30)$

\section{DISCUSSION}

The aim of this study was to collect information regarding the presentations, physical findings, diagnostic findings complications the patients with chronic calcific pancreatitis. In our series the youngest was of 16 years and oldest was of 65 years old. Pancreatic calculi is said to be more common in male, in our series male to female ratio is $10: 1$. In the first large series of 54 cases of pancreatic calculi by Zuidema [8] from Indonesia, 31 patients were below the age of 31 years. In the Nigerian Series by Kinner 47 out of 30 cases of pancreatic calculi, 26 were below the age of 29 years in 1963. In our series the average age of the ten patients is also 43 years. According to Rodney [7] smith pancreatitis is a rare disease in England when compared to the United States. He is of the opinion that alcoholic and calcific pancreatitis is not common in North-west England, possibly owing to the low incidence of heavy alcoholism in that country. In 1963 Fitz Gerald et al. reported 53 cases of pancreatitis studied during a period of 8 years from Ireland. They emphasis the importance of sphincteric stenosis, a rarity of alcoholism among the causative factors. The rarity of chronic relapsing pancreatitis in Israel is thought to be due to the religious prohibition of alcohol in that country. The low incidence of alcoholic pancreatitis reported from Czechoslovakia is ascribed to consumption of diluted liquor by the population in general. The high incidence of pancreatitis in U.S.A. and in some European Countries are distinctly related to alcoholism. The high incidence of pancreatitis in countries where alcoholism in rare is attributed to protein malnutrition, first suggested by Zuidema from Indonesia, later supported by Shaper from Uganda, Kinner from Nigeria. As compared with the western series, nine patients $(30 \%)$ were alcoholic who presented with other hepatobiliary tract disease. 16 patients $(53.33 \%)$ were smoker. The probable causes of pancreatitis in our patients may be protein malnutrition, parasitic infestations and familial pancreatitis. Zuidema and Shaper stressed the importance of protein malnutrition in the causation of pancreatic fibrosis in juvenile diabetes with calcification. As the daily turnover of protein in the pancreatic juice is just second to the intestine, the pancreas suffers much in protein deficiency and leads to degeneration and fibrosis of the acini and metaplasia of the ductal epithelium. In support of this theory, Howard and Jordan showed the incidence of cirrhosis of liver in cases of pancreatic calculi is quite high, about 47\%. According to Howat in alcoholic pancreatitis with adequate protein intake cirrhosis of liver was virtually absent. Bilateral parotid enlargement is evidence in favor of protein mal-nutrition in the series of zuindema $30 \%$ cases and in Shapher's series $42 \%$ cases had bilateral parotitis without pain, fever, discharge of pas from the stensen's duct. It is a compensatory mechanism for the pancreatic deficiency 
because parotid biopsy is found normal and the pancreas is said to be the 'abdominal salivary gland'. In our series significant protein deficiency could not be found out, as all the patients was one average Bengali diet, nobody was vegetarian, which contained about 500 gm boiled rice, 50-75 gm of fish, occasional meat, eggs and about $30 \mathrm{gm}$ of mustard oil, Soybean oil yielding about 2000 calories daily. If protein malnutrition be the case of pancreatic calculi, all the Kwashiorkor patients would develop it, which is not found. Parasitic infestations may play the major role in the causation of chronic pancreatitis with calcification in the developing countries, where the environmental sanitation is very poor and almost every person have suffered from Various parasitic infestations at least once in the life. In this part of the World, biliary obstruction and cholecystitis in association with round 64 reported 11 cases of pancreatitis occurring in children and observed that round worm infestation was an important cause. Mention has been made about a number of cases of hydatid disease of the pancreas occurring in North Africa [9]. Tong-That-Tung et al. [10] have reported 189 cases of pancreatitis in adults caused by ascaris infestations of the common bile duct in Vietnam. Chiung [11] observed 141 cases of biliary ascaris in 6 years form China. In our series $30 \%$ cases has either ova of ascaris lumbricoids or cysts of giardia or cysts of E. histolytica are found in the routine examination of the stool and high eosinophil count in $20 \%$ cases. Familial pancreatitis can't be ruled out as special investigation regarding amino aciduria or Sweat test, etc. we're not performed. One patient gave the family history of abdominal pain of her parents, though not detailed operative finding were available according to her statement her mother was operated for it, probably any operative procedure for peptic ulcer was done. No patient gave any history of trauma to the abdomen, mumps and steroid therapy. No patient showed hypercalcemia or hypercalcemia or hyperlipidemia. $40 \%$ patients of our series were belonged to ' $\mathrm{O}$ ' group $30 \%$ of ' $A$ ' group, $10 \%$ of ' $A B$ ' and ' $\mathrm{B}$ ' group. Of the five types of clinical presentations of chronic and relapsing chronic pancreatitis, three types were found in the series. Upper abdominal pain was present in all thirty cases, Diabetes mellitus was present in six cases and nine cases was presented as with a tenderness in the right hypochondrium three of them who subsequently developed obstructive jaundice. Eight cases presented with malabsorption syndrome or steatorrhoea. Abdominal pain was typical of pancreatic pain developed in the epigastrium passing through to the back, which usually started about one hour after the meals, improved in sitting position and aggravated in lying condition continuing for days together relentlessly not responding to usual analgesic or antispasmodics, in majority of cases necessitating hospital or clinic admission. The pain was recurrent in twenty patients who developed the pain at intervals of few months to four years. In eight cases, in which cases vomiting was also present. In six cases, the pain was continuous for about six or seven months which bound them to get admitted for operative treatment into the hospital. Three patient had smokers $(55 \%)$ while Zuidema 45 reported $30 \%$ and shaper $6942 \%$ of their cases had it in the large series of Geevarghees [7] only two cases out of 600 cases had smoker. Complication following surgery were persistence of the fistulous tract following removal of the drain tube in once case which was sealed spontaneously after regular dressing within three weeks of operation, Subacute intestinal obstruction in one case which was also improved by conservative treatment, Respiratory tract infection in two cases and wound infection in two case which were also improved after appropriate antibiotic therapy. One patient developed post-operative Hepatitis B viral infection about two months after the operation and suffered for one month. One patient developed recurrent pain with jaundice but improved after conservative management. The management of $\mathrm{CP}$ is challenging and requires a personalized approach focused on the individual patient's main symptoms, goals, and quality of life [12]. Most patients remain symptomatic despite therapy [13]. Most patients with $\mathrm{CP}$ have abdominal pain, with a reported prevalence of $50-85 \%[14,15]$. Pain in CP is multifactorial, with inflammatory and neuropathic components [16]. The pain in the past was believed to be primarily due to obstruction of the pancreatic duct by either stricture or stone, resulting in high pressure and ischemia above the obstruction [13]. Now a day, genetic factors are also considered as potential issues in the discussion of chronic pancreatitis. But in this study we did not consider it. CP is now recognized as a complex disease with multiple associated genetic risk factors and disease modifiers [17]. Genetic variations strongly associated with $\mathrm{CP}$ are those in PRSS1 (cationic trypsinogen), SPINK1 (serine protease inhibitor kazaltype 1), and CFTR (cystic fibrosis transmembrane conductance regulator) and, to a lesser extent, CTRC (chymotrypsin C) and CASR (calcium-sensing receptor) [17].

These mutations and polymorphisms have different mechanisms and variable penetrance. The most potent mutation is in PRSS1, a gain-of-function mutation, which can cause the autosomal dominant condition of hereditary pancreatitis (HP) [18]. HP differs from many other forms of pancreatitis in the early onset, rapid progression to end-stage $\mathrm{CP}$, and a significantly increased risk of pancreatic adenocarcinoma [18]. Mutations and polymorphisms in other genes function as risk factors and disease modifiers. Genetic testing might be considered when patients have a family history of idiopathic CP, RAP, or childhood pancreatitis, have relatives with known mutations associated with HP, are younger than 25 years old, or have RAP of uncertain etiology [18]. 


\section{CONCLUSION AND}

\section{RECOMMENDATIONS}

In this study, weight loss and diabetes were two major clinical presentations among the participants. This study carries some epidemiological significance in certain aspects. Persistent or recurrent pain is the most common presentation in this study. In diagnosis history of pain, radiology and Ultrasonography were used. CT scan and ERCP is the second line investigation in the diagnostic procedures. If indicated most of the cases are operable and drainage procedure (Puestow procedure) is the best option. Surgery is a standard form of treatment and outcome of surgery is quite appreciable in our country except few cases which histologically proved malignant postoperatively and later was treated by chemotherapy. For getting more specific findings we would like to recommend for conducting more studies regarding the same issue with larger sized sample.

\section{REFERENCES}

1. William F.Ganong. 'Review of Medical Physiology' 11th Ed. C1983Lange Medical Publications California, USA. P. 399-401, 268, 271-286.

2. Davis-Chistopher Text book of surgery-12th Ed. (C) 1981 by W.B. Saunders Company, Tokyo.1283.

3. Samson Wright's Applied Physiology-13th Ed.@ Oxford university Press, 1982, London P. 4301,503-8,432.

4. Hermann, Robert E. Mannual Of surgery of gail biadder, Bile ducts and exocrine pancreas. (C) 1979 by springer-verlar p. 177 .

5. Pathologic Basis of Disease Robins, Stanley L. et 3rd Ed. (C) 1984 W.B. Saunders Company. 963-7.

6. WHO Expert Commette on Diabetes mellitus Technical Report Series 646, 1980; 23.
7. Geevarghese PJ. "Pancreatic Diabetes"- A Clinico Pathologic Study of Growth Onset Diabetes with Pancrectic Calculi" Popular Prokashan, Bombay, 1968.

8. Zuidema PJ. "Cirrhosis and disseminated calcification of the pancreas in patients with malnutrition". Trop. and Geography. Med. ii: 70, 1959.

9. Hess W. "Surgery of the biliary passages \& the pancreas translated from the German by Heinrich Lamm. V.Van. Nostrand Company. Inc. Princeton, New Jersey, 1965; 89.

10. Tong-That-Tung, Nguyen-Duong-Ouang \& Hoang Kim-Tinh:- Vestn. Khir. 1960; 84:3.

11. Chiung, WH. "Biliary ascariasis, an analysis of 141 cases" Chin. Med. J. 1956; 74:445.

12. Muniraj T, Aslanian HR, Farrell J. Chronic pancreatitis, a comprehensive review and update. Part II: Diagnosis, complications, and management. Dis Mon. 2015; 61(1): 5-37.

13. Forsmark CE. Management of chronic pancreatitis. Gastroenterology. 2013; 144(6): 1282-91.e3.

14. Ammann RW, Muellhaupt B. The natural history of pain in alcoholic chronic pancreatitis. Gastroenterology. 1999; 116(5): 1132-40.

15. Fasanella KE, Davis B, Lyons J. Pain in chronic pancreatitis and pancreatic cancer. Gastroenterol Clin North Am. 2007; 36(2): 335-64.

16. Gupte AR, Forsmark CE: Chronic pancreatitis. Curr Opin Gastroenterol. 2014; 30(5): 500-5.

17. Muniraj T, Aslanian HR, Farrell J. Chronic pancreatitis, a comprehensive review and update. Part I: epidemiology, etiology, risk factors, genetics, pathophysiology, and clinical features. Dis Mon. 2014; 60(12): 530-50.

18. Ammann R. Endoscopic duct obstruction--is it an alternative to surgery for pain relief in chronic pancreatitis? Gastrointest Endosc. 1981; 27(2): 106-7. 